\title{
EVALUATION OF CUTTING EFFICIENCY AND SURFACE CHARACTERISTICS OF XP ENDO SHAPER AND IRACE NITI ROTARY ENDODONTIC INSTRUMENTS (A COMPARATIVE IN VITRO STUDY)
}

Samah Abdelrahim Salih*, Siza Yacoub Zakhary** and Marwa Mahmoud Bedier***

\begin{abstract}
Aim: The aim of the present study was to compare the cutting efficiency of XP-Endo shaper and $\mathrm{iRaCe}$ nickel titanium rotary instruments after different numbers of uses and evaluate the surface characteristics.

Methodology: Forty-eight mesial roots were divided into two groups ( $n=24)$ according to the type of the file system used (XPS/iRaCe) and was then subdivided into three subgroups $(n=8)$ according to the number of uses (first, second and third uses). Each specimen was prepared and irrigation was done with $5 \mathrm{ml}$ of freshly prepared $2.5 \%$ NOCL, then $3 \mathrm{ml}$ of $17 \%$ EDTA followed by $10 \mathrm{ml}$ of distilled water. All root samples were weighed before and after preparation and weight loss was calculated. Two samples (Size 30 and Taper 0.04) from each file system were randomly selected and photographed with the Scanning Electron Microscope (SEM) before and after the third use, at magnification of 150x and 2000x. Comparing the cutting efficiency of each group at the different numbers of uses was done with analysis of variance (ANOVA) and multiple comparisons post hoc test.
\end{abstract}

Results: There was a statistically significant difference between XPS and iRaCe after each use $(\mathrm{P} \leq 0.05)$, with drop in the cutting efficiency in both groups after the second and third uses, and no statistical difference between second and third uses ( $P>0.001)$. SEM examination of unused files showed manufactural defects, while files after third use showed other defects.

Conclusions: XPS showed higher cutting efficiency than iRaCe, with reduction in its cutting efficiency after multiple use.

KEYWORDS: Cutting efficiency; Defects; iRaCe; SEM; XPS.

\footnotetext{
* Endodontics, Faculty of Dentistry, Cairo University

** Professor of Endodontics Faculty of Dentistry Cairo University

*** Associate Professor of Endodontics Faculty of Dentistry Cairo University
} 


\section{INTRODUCTION}

The main goal of root canal therapy is the elimination of microorganisms and their by-products from the root canal system and providing a tight seal to prevent reinfection, thus a combination of mechanical instrumentation and irrigating solutions has been used to remove the organic debris and to eliminate the microorganisms and its by products ${ }^{(1)}$.

Different instruments designs play an important role in cutting efficiency. Cutting ability is the ability of the instruments to cut through a given material, while the cutting efficiency is the rate of the cut of the instrument through a given material. The cutting ability and cleaning efficiency depend on different parameters such as; the metallurgical properties, surface treatment of the instrument, cross sectional configuration of the shaft, sharpness of flute, flute design (number of flutes, helical angle, and rake angle) tip design. Other factors that can also play a role; lubrication during cutting, wear resistance, chip removal capability, sterilization and mode of use $^{(2,3)}$

Several studies reported that the presence of defects such as metal flashes, microcavities, scratches and crack allow the instruments to be more prone to fracture, in addition to reducing the cutting efficiency of endodontic instruments. Machining of NiTi instruments may be responsible for the production of manufacturing defects on the instrument surface, where they act as points of tension capable of initiating fracture ${ }^{(4)}$.

iRaCe (FKG, La Chaux-de-Fonds, Switzerland) NiTi rotary files have been introduced as a simplified sequence of the RaCe NiTi rotary files (FKG) with sharp cutting edges to improve cutting efficiency and alternating cutting edges to eliminate screwing ${ }^{(5)}$. Although they have been subject to electropolishing, they still have machining grooves left on the surface after the manufacturing process which might be an initiator of a microcrack ${ }^{(6)}$.

Recently the XP-Endo Shaper (XPS) manufactured from MaxWire alloy has been introduced, which allows the instrument to change its phase according to the changes in temperature. The XPS is in a martensitic phase; however, it changes to an austenitic phase when placed inside the canal at body temperature ${ }^{(8)}$. At the body temperature within canals, the XPS expands and contracts to adapt itself to the canal morphology, therefore it leaves fewer untouched walls, applies minimal stress on the dentin walls, thereby minimizing the risk of microcracks in the dentin ${ }^{(9)}$.

Up to date no study has compared the cutting efficiency and surface characteristics of XP-Endo shaper rotary system with iRace. Thus the purpose of this study was to compare the cutting efficiency of XP-Endo shaper and iRaCe nickel titanium rotary instruments and to evaluate the surface characteristics by scanning electron microscope (SEM). The null hypothesis was that there would be no statistically significant difference between XP-Endo shaper and $\mathrm{iRaCe}$ regarding the cutting efficiency.

\section{MATERIALS AND METHODS}

\section{Sample size calculation}

Sample size calculation was based upon the results of Kataia et al. $2016^{(10)}$, utilizing the change in weight as the primary outcome, using alpha $(\alpha)$ level of $0.05(5 \%)$ and Beta $(\beta)$ level of $0.20(20 \%)$ i.e. power $=80 \%$ and effect size $8.8 \times 104 \mathrm{gm}$ is expected. The minimum estimated sample size was 24 samples per group and 8 teeth in each subgroub. Sample size calculation was performed using PS; Power and Sample size calculation software Version 3.1.2 (Vanderbilt University, Nashville, Tennessee, USA).

\section{Selection of the Samples}

Intact recently extracted forty eight mandibular molars due to periodontal disease with fully formed apices were collected from Oral and Maxillofacial surgery department, Faculty of Dentistry, Cairo University. The protocol for this comparative in vitro designed trial was reviewed and approved by 
the ethics committee, Faculty of Dentistry, Cairo University, with respect to scientific content and compliance with applicable research and human subjects' regulations.

All teeth were examined to exclude those with calcified root canals, root caries, root resorption, cracks, and previous endodontic treatment. Preoperative radiograph was taken to ensure no calcification and to measure root canal curvature. Teeth with root curvature $25-35^{\circ}$ with an average of $27^{\circ}$ and tooth length of $20 \pm 1$ was chosen and included in the study. Teeth were thoroughly washed under running water, then the root surfaces were planed and any soft tissue remnants or calculus were removed from the root surfaces using the ultrasonic scaler. The teeth were then stored in saline at room temperature till the time of use.

\section{Preparation of the Samples}

Access cavity preparation was done using round diamond bur size \# 3 and Endo-Z bur, then the canals were checked for apical patency using size 15 stainless-steel $\mathrm{K}$ file in each canal until visible at the apical foramen. Roots with apical foramen size more than 20 or less than 15 were excluded and the working length was measured by measuring the tooth length and subtracting $1 \mathrm{~mm}$.

All teeth were hemi-sectioned bucco-lingually at the furcation level with a diamond disc, mounted in high speed contra-angle under copious water coolant, the distal root with its part of the crown was discarded and the mesial half was used for the preparation.

\section{Grouping of the Samples}

Forty eight mesial roots were used. Samples were divided into two groups $(n=24)$ according to the type of the file system used; XPS /iRaCe (FKG Dentaire, Switzerland). Each group was then subdivided into three subgroups $(n=8)$ according to the number of uses of the rotary system (first use F, second use $\mathrm{S}$ and third use $\mathrm{T})$. Random sequence was generated by using random sequence generator website and allocation concealment was achieved using sequentially numbered (1 to 48 ) opaque sealed envelope.

\section{Root canal preparation of the samples}

In Group (XPS); each root canal was flushed with freshly prepared $5 \mathrm{ml}$ of $2.5 \% \mathrm{NaOCl}$ using 30 gauge side vented needle (NaviTip, Ultradent South Jordan, UT, US), then XPS (size 30 and taper 0.04) was mounted on an X-Smart plus motor (Dentsply, Maillefer, Switzerland) and inserted into the canal at speed $800 \mathrm{rpm}$ and torque $1 \mathrm{Ncm}$.

Five gentle strokes were applied (in-and-out motion) until the working length was reached. In cases where the instrument failed to reach the working length, the canals were irrigated again with $5 \mathrm{ml}$ of $2.5 \% \mathrm{NaOCl}$, recapitulated with size 15 stainless-steel K-file and then re-entered again with the XPS. Once the file reached the full working length, 10 more up-and-down movements were done with the XPS file.At the end of the preparation, $5 \mathrm{ml}$ of $17 \%$ EDTA was used for $1 \mathrm{~min}$ to remove the smear layer followed by $10 \mathrm{ml}$ distilled water as final flush to prevent erosion of the dentinal tubules.

In Group (iRaCe); R1 file (size 25 and taper 0.04) was mounted on the motor adjusted at speed of 600 rpm and torque $1.5 \mathrm{Ncm}$ to prepare the coronal and the middle third of the canal, then R2 (size 25 and taper 0.04) was used to the full working length, and finally, R3 (size 30 and taper 0.04) was used to prepare the samples to the full working length with the same irrigation protocol.

\section{Evaluation of the cutting efficiency}

Before preparation all root samples were left to dry in open air for 24-hrs, then labeled with serial number and weighed using the four- digit gram high precision balance sensitive scale (Scaltec SBC, Germany). After root canal preparation, the root samples were dried with the corresponding paper point and left to dry in open air for 24-hrs, 
then each sample was re-weighed using the fourdigit gram high precision balance sensitive scale. Measurements were recorded in a chart and repeated three times for reproducibility.

Weight loss was calculated through measuring the difference between the weight before preparation and the weight after preparation (Wt. Before - Wt. After), which was used to compare the cutting efficiency of the two systems.

\section{Evaluation of the Surface characteristics}

Two representative samples (Size 30 and Taper 0.04) from each file system were randomly selected and photographed with the Scanning Electron Microscope (Hitachi S-4800-II FESEM, California,USA) before use and after the third use, at magnification of $150 x$ and 2000x respectively.

All files were cleaned in an ultrasonic cleaner using ethyl alcohol for 25 minutes to remove the debris, and then 2 representative samples from each group were scanned. In an attempt to evaluate the same area of a file on multiple examinations, a line was drawn with a permanent ink on the handle of the file at the evaluated side, then the middle and apical thirds of the file were photographed.

Files were examined for the presence of microfracture, complete fracture, cracks, metal strips, metal rollover, milling marks, unwinding of the flutes, scratches, pitting, corrosion and debris adhering to the files surface even after ultrasonic cleaning.

\section{Statistical Analysis of the Data}

Numerical data were explored for normality by checking the distribution of data and using tests of normality (Kolmogorov-Smirnov and ShapiroWilk tests). All data showed normal (parametric) distribution. Parametric Data were presented as means and standard deviations (SD). Comparison between the two groups after each use was done using the Unpaired t test, while analysis of variance (ANOVA) was used to study the effect of number of uses on the cutting efficiency of each group.
Post-hoc test was used for pair-wise comparisons when ANOVA test was significant. The significance level was set at $\mathrm{P} \leq 0.05$. Statistical analysis was performed with IBM SPSS (IBM Corporation, NY, USA) Statistics Version 20 for Windows.

\section{RESULTS}

Results showed statistically significant difference in the cutting efficiency after different numbers of uses $(\mathrm{P}=0.001, \mathrm{P} \leq 0.05)$. Group (XPS), (XPS-F) showed the highest weight loss followed by (XPS-S) and (XPS-T) and in Group (iRaCe): (iRace-F) showed the highest weight loss followed by (iRaCe-S) and (iRaCe-T).Comparing the cutting efficiency of the two file systems at each number of use, result showed statistically significant difference between XPS and $\mathrm{iRaCe}(\mathrm{P}=0.001, \mathrm{P} \leq 0.05)$, where the XPS showed greater weight loss than iRaCe in all number of uses.

At magnification of 150x, XPS and iRace showed milling marks and metal flashes with sharp cutting edges before use and at magnification of 2000x milling marks, metal flashes and pits were detected. After the third use, XPS and iRaCe showed debris, milling marks, pits and blunting of cutting edge at magnification of $150 x$, while at magnification of 2000x, debris, scratches, pits, milling marks and cracks were detected.

\section{DISCUSSION}

The main objectives of root canal instrumentation is shaping and cleaning the root canal system effectively while maintaining the original canal configuration ${ }^{(11)}$. Canal preparation involves elimination of necrotic tissue, debris and shaping of root canals without changing the primary shape of the canal ${ }^{(12)}$. NiTi rotary instruments had undergone revolutionary changes in terms of the methods of fabrication and hence their physical properties. Moreover, the design, shape, and number of instruments used have highly been modified ${ }^{(13)}$. The aim of modifying and improving of these instruments 
is to develop a NiTi rotary instrument which resist fracture even in most challenging narrow curved root canals, to simplify the cleaning and shaping stage and to reduce the number of instruments used while preserving the original shape of the prepared root canals, in addition to cut and remove the dentin efficiently ${ }^{(14)}$.

The cutting capacity of NiTi rotary instruments is related to the complex inter-relationship between different parameters such as the sectional design of the instruments, radial lands or active cutting blades, metallurgical properties, and treatment of the instrument surfaces with the incorporation of ions ${ }^{(15)}$. New NiTi endodontic files with superior properties have been developed with special thermomechanical processing, to improve their flexibility, mechanical behavior and to give the instruments better flexural fatigue resistance than files made from conventional NiTi alloy ${ }^{(16)}$.

$i R a C e$ Nickel titanium rotary endodontic instruments have been introduced as a simplified sequence of the RaCe system, manufactured from a conventional austenitic NiTi alloy which is strong, hard and less flexible, with an electropolished surface ${ }^{(5)}$ and recently the XP-Endo Shaper (XPS) manufactured from MaxWire alloy has been introduced, which allows the instrument to change its phase according to the changes in temperature ${ }^{(7)}$.

Literature has reported several methods for measuring the cutting efficiency; such as changes in the dentin thickness and root canal volume using multi-slice computed topography, volume of bovine bone removed per unit time, measuring the depth of cut in bovine bone or polymethylmethacrylate samples, weight loss using sensitive scale or calculating the surface area of cut grooves, in addition to measuring the maximum penetration depth of the instruments into the lumen of special plastic samples with a cylindrical canal ${ }^{(17-20)}$. In the present study measuring the mean sample weight loss before and after using digital scale was used for measuring the cutting efficiency of the two systems was done in accordance to Vinothkumar et al. 2007(19), Cecchin et al. 2011 (21), Sergio et al. $2012^{(22)}$, Kataia et al $.2016^{(10)}$ as mean weight loss is one of the simplest and most precise methods to measure the extracted volume of dentin.

Variable materials were used as substrate to measure the cutting efficiency of the instruments such as, the extracted teeth, acrylic wafer, acrylic blocks, Plexiglas and bovine bone ${ }^{(23,24)}$.Although the use of Plexiglas allows different instruments to be tested on identical samples, eliminate variations in hardness between samples that may influence results, it does not exhibit the same properties as dentin and thus does not provide clinical relevant data ${ }^{(23)}$. In addition, the use of bovine bone specimens allow standardization in the shape and orientation, as well as exhibiting comparable hardness to dentin, but still cutting into bone does not reproduce the action of instrumentation in a root canal ${ }^{(25)}$.

Thus, in the present study extracted teeth were used in accordance to Vinothkumar et al. $2007^{(19)}$, Sergio et al. $2012^{(22)}$ kataia et et al. $2016^{(10)}$, where extracted teeth gave the study greater reliability being more similar to clinical conditions than artificial canals made of acrylic resin, in addition, they reflect the action of the instruments in clinical use ${ }^{(26,27)}$.

Results of the present study showed that XPS has higher cutting efficiency than iRaCe rotary system, which was in consistency with the results of Azim et al. 2017(28) who stated that XPS left fewer untouched walls (38.6\%) than Vortex Blue $(58.8 \%)$. This may be attributed to the difference in the alloy used for manufacturing the files; where the $\mathrm{iRaCe}$ is manufactured from a conventional austenitic NiTi alloy which is strong and hard (5) , while the innovative XPS manufactured from a highly flexible alloy (MaxWire), once subjected to body temperature the alloy undergoes a phase transformation shifting from the martensitic which is soft, ductile and can be easily deformed to the austenitic phase; which allows the file to expand 
beyond its core size and address more canal walls, hence, the name "core adaptive file" ${ }^{(29)}$ Moreover, XPS has a smaller core and attempts to "adapt" to the canal walls, to reach areas that conventional instruments cannot access, therefore leaving less untouched canal area and, at the same time perform three dimensions canal shaping ${ }^{(19)}$.

The result was in contrast to, Versiani et al. $2017^{(30)}$ who revealed similar shaping ability of XP shaper and $\mathrm{iRaCe}$ by measuring volume change in dentin using micro computed tomography. Zuolo et al. $2018^{(31)}$ reported similar shaping of curved canal with self-adjusting file and BioRaCe.These differences across studies may be attributed to variations in methodological models such as tooth type, instrumentation duration, irrigation amount and method ${ }^{(30)}$.

The cutting efficiency of both files decreased after repeated use, where the first use showed higher cutting efficiency than the second and the third use, with no difference between the second and third use. The reduction in the cutting efficiency of the instruments could be attributed to modifications of the cutting edges, this was in agreement with the results of Svec et al. $2002{ }^{(32)}$ revealed evidence of flaking, pitting and crack formation on the surface of rotary instrument after repeated use , Xi Wei et al. $2007^{(33)}$ reported signs of wear and roll over of the cutting edges of the instruments, where these modifications of the cutting edges adversely affect the cutting efficiency.

Scanning electron microscope (SEM) was used to evaluate the surface characteristics of the instruments in the current study, no visible defects, no microfracture or cracks were observed in the before use group, while manufacturing landmarks as milling marks and metal flashes were detected, this was in agreement with Alexandrou et al. 2006 ${ }^{(34)}$ and Condorelli et al. (2010) ${ }^{(35)}$. These results indicate that the manufacturing process and packing conditions of rotary endodontic files are far from ideal ${ }^{(36)}$.
Examination of the instruments after third use with scanning electron microscope showed various amounts of machining grooves, metal flash, debris, scratches, pits, blunting of the cutting edge and cracks. This was in agreement with Kaul et al. 2014 (37) who found notches and wear of flute edges of Race rotary file after preparation of seven canals. The blunt edges observed were produced by friction of the instruments against the root canal walls, while the formation of metal flashes and pitting on surface of NiTi files after use is the result of corrosion and deterioration of NiTi instruments during instrumentation in the presence of $\mathrm{NaOCl}$ and EDTA ${ }^{(38,39)}$. These defects reduce the cutting efficiency of the rotary files and increase the liability to fracture.

In conclusion, XP-Endo shaper has higher cutting efficiency than iRaCe nickel titanium rotary endodontic instruments, XP-Endo shaper and iRaCe showed reduction in the cutting efficiency of instruments after multiple uses and examination of the instruments before and after third use with SEM showed varying amounts of defects.

The authors declare that they have no conflict of interest.

\section{REFRENECES}

1. Neves MA, Provenzano JC. Clinical antibacterial effectiveness of root canal preparation with reciprocating single-instrument or continuously rotating multi-instrument systems. J Endod 2016; 42: 25- 29.

2. Henry SY and Von Stebut J. Cutting effieciency loss of root canal instruments due to bulk plastic transformation. J Endod 1994; 20: 367-72.

3. Felt RA, Moser JB and Heuer MA: Flute design of endodontic instruments and its influence on the cutting efficiency. J Endod 1982; 8: 253-259.

4. Berutti E, Angelini E, Rigolone M, Migliaretti G, Pasqualini D. Influence of sodium hypochlorite on fracture properties and corrosion of ProTaper rotary instruments. Int Endod J 2006; 39: 693-699.

5. Saber SE, Nagy MM, Schafer E. Comparative evaluation of the shaping ability of ProTaper Next, iRaCe and hyflex $\mathrm{CM}$ rotary NiTi files in severly curved root canals. Int Endod J 2015; 48: 131-136. 
6. Wu H, Peng C, Bai Y, Hu X, Wang L. Shaping ability of ProTaper Universal, WaveOne and ProTaper Next in simulated L- shaped and S-shaped root canals. BMC Oral Health 2015; 15: 27.

7. Silva EJ, Vieira VT, Belladonna FG, Zuolo AS, Antunes HD, Cavalcante DM, Elias CN, De-Deus G. Cyclic and torsional fatigue resistance of XP-endo Shaper and TRUShape instruments. J Endod 2018; 44: 168-172.

8. Elnaghy AM, Elsaka SE. Torsional resistance of XP-endo Shaper at body temperature compared with several nickel titanium rotary instruments. Int Endo J 2017; 27: 1111-12815.

9. Bayram HM, Bayram E, Ocak M, Uygun AD, Celik HH. Effect of ProTaper Gold, self-adjusting File, and XP-endo Shaper instruments on dentinal microcrack formation: a micro-computed tomographic study. J Endod 2017; 43: 1166-1169.

10. Kataia, M M, Kataia, EM. Cutting efficiency of different cross sectional design ProTaper rotary instruments - invitro study. JIPBS. 2016; 3: 116-122.

11. Siqueira JF Jr. Endodontic infections: concepts, paradigms, and perspectives. Oral Surg Oral Med Oral Pathol Oral Radiol Endod 2002; 94: 281-293.

12. Bergmans L, Cleynenbreugel JV, Wevers M, Lambrechts P. Mechanical root canal preparation with NiTi rotary instruments: Rationale, performance and safety. Am J Dent 2001; 324-233.

13. Hulsmann M, Peters O, Dummer PMH. Mechanical preparation of root canals, shaping goals, techniques and means. Endod topics 2005; 10: 30-76.

14. Schneider SW: A comparison of canal preparation in straight and curved canals. Oral Surg 1971; 32: 271-275.

15. Felt RA, Moser JB and Heuer MA: Flute design of endodontic instruments \& its influence on the cutting efficiency. J Endod 1982. 8: 253-259.

16. Guelzow A, Stamm P, Kielbassa M. Comparative study of six rotary nickel titanium systems and hand instrumentation of root canal preparation. Int Endod J 2005; 38:743-752.

17. Fayyad DM, Elhakim E. Cutting efficiency of twisted versus machined nickel titanium Endodontic files. J Endod 2011; 37: 1143-1146.

18. Machian G, Peters D, Lorton L.The comparative efficiency of four types of endodontic instruments. J Endod 1982; 8: 398-402.
19. Vinothkumar TS, Miglani R. Influence of Deep Dry Cryogenic Treatment on Cutting Efficiency and Wear Resistance of Nickel-Titanium Rotary Endodontic Instruments. J Endod 2007; 33: 1355-1358.

20. Schafer E, Oitzinger M. Cutting efficiency of five different types of rotary nickel-titanium instruments. J Endod 2008; 34: 198-200.

21. Cecchin D, de Sousa-Neto MD, Pecora JD. Cutting efficiency of four different rotary nickel titanium instruments. J Conserv Dent 2011; 14: 117-119.

22. Sergio H, Marcelo G, Mario T. Cutting ability of nickeltitanium rotary systems ProTaper, Mtwo and K3. RSBO 2012; 9: 177-182.

23. Gianluca P, Alessio G, Nicola M. Cutting Efficiency of Reciproc and WaveOne Reciprocating Instruments. J Endod 2014; 40: 1228-1230.

24. Stenman E and Spangberg LSW: Machining efficiency of endodontic files a new methodology. J Endod 1990; 16: 151-157.

25. Haikel Y, Serfaty R, Lwin TT. Measyrment of the cutting efficiency of endodontic instruments: a new concept . J Endod 1996; 12: 651-656.

26. Al-Omari MA, Dummer PM, Newcombe RG. Comparsion of six files to prepare simulated root canals. Int Endod J 1992; 25: 67-81.

27. Yun HH, Kim SK. A Comparison of the shaping abilities of 4 nickel-titanium rotary instruments in simulated root canals. Oral Surg Oral Med Oral Pathol Oral Radiol Endod 2003; 95: 228-233.

28. Azim AA, Aksel H, Zhuang T, Mashtare T, Babu JP, Huang GT. Efficacy of 4 irrigation protocols in killing bacteria colonized in dentinal tubules examined by a novel confocal laser scanning microscope analysis. J Endod 2016; 42: 928-934.

29. Paqué F, Balmer M, Attin T, Peters OA. Preparation of oval-shaped root canals in mandibular molars using nickel-titanium rotary instruments: a micro-computed tomography study. J Endod 2010 ; 36: 703-707.

30. Versiani MA, Carvalho. Micro-computed tomographic evaluation of the shaping ability of XP-endo shaper, iRace andEdgeFile systems in long oval-shaped canals. J Endod 2018; 44: 489-495. 
31. Zuolo M, Zaia A, Belladonna F. Micro-CT assessment of the shaping ability of four root canal instrumentation systems in oval-shaped canal. Int Endod J 2018; 51: 564 -571.

32. Svec T, Powers J. The Deterioration of Rotary Nickel-Titanium Files under Controlled Conditions. J Endod 2002; 28: 105-107.

33. Xi Wei, Junqi L, Jin J. Modes of failure of ProTaper nickel0titanium rotary instruments after clinical use. J Endod 2007; 3: 276-279.

34. Alexandrou GB, Chrissafis K, Vasiliadis LP. SEM observations and differential scanning calorimetric studies of new and sterilized nickel-titanium rotary endodontic instruments. J Endod 2006; 7: 675-679.

35. Condorelli G, Bonaccorso A, Smecca E. Improvement of the fatigue resistance of NiTi endodontic files by surface and bulk modifications. Int Endod J 2010; 10: 866-873.
36. Kaul R, Farooq R. Comparative Evaluation of Physical Surface Changes and Incidence of Separation in Rotary Nickel Titanium Instruments: An in vitro SEM study. IEJ 2014; 9: 204-209.

37. Elemam R, Capelas J, Vieira M. Effect of repeated use on topographical features of ProTaper Next Endodontic Rotary File. J Int Oral Health 2016; 8: 445-450.

38. Caballero H, River F, Salas H. Scanning electron microscopy of superficical defects in twisted files and reciproc nickel titanium files after use in extracted molars. Int Endod J 2015; 48: 229-235.

39. Cai JJ, Tang XN, Ge JY. Effect of irrigation on surface roughness and fatigue resistance of controlled memory wire nickel titanium instruments. Int Endod J 2017; 50: 718-724. 